Didáctica. Lengua y literatura

ISSN-e: 1988-2548

https://dx.doi.org/10.5209/dida.56932

\title{
Genius Hour: una estrategia metodológica innovadora para la clase de Español como Lengua Extranjera
}

\author{
Clara Bieger Morales ${ }^{1}$; Presentación Caballero-García ${ }^{2}$
}

Recibido: 4 de agosto de 2017 / Aceptado: 5 de marzo de 2019

Resumen. La propuesta de innovación educativa que se presenta en este trabajo, llamada Genius Hour, es una aportación del mundo empresarial que queremos utilizar como estrategia de aprendizaje innovadora en la clase de Español como Lengua Extranjera (ELE). Genius Hour se encuadra dentro la metodología del aprendizaje basado en proyectos (ABP), en el que la investigación juega un papel fundamental. Está pensada para ser implementada en un contexto escolar multicultural de alumnos adolescentes. Cuenta aspectos básicos de cómo puede ser desarrollada en el aula de ELE, beneficios esperados a partir de la fundamentación teórica y la experiencia profesional, y termina señalando sus vías de continuidad, entre las que destacamos la contrastación empírica de esta experiencia exitosa de empresa y de cambio propuesto para la enseñanza del español.

Palabras clave: Clase de ELE, Genius Hour, innovación educativa, Aprendizaje basado en la Investigación (ABI), Aprendizaje basado en Proyectos (ABP).

[en] Genius Hour: an innovative methodological strategy in the Spanish as a Foreign Language class

\begin{abstract}
This work proposes to use Genius Hour, an idea that originated in a business environment, as an innovative teaching and learning strategy in the Spanish as a Second Language (SSL) class. Genius hour falls within the category of Project-Based Learning (PBL), where inquiry plays a fundamental role. This work will focus on a multicultural secondary school context. Throughout it, basic aspects on how to implement this strategy are described, as well as its expected pedagogical benefits. Lastly, possible future work ideas are presented. We suggest carrying out an empirical study to confirm the theoretical outcomes predicted by this proposal.
\end{abstract}

Keywords: Spanish as a Second Language (SSL) class, Genius Hour, pedagogical innovation, InquiryBased Learning (IBL), Project-Based Learning (PBL).

\section{[fr] Genius Hour: une stratégie méthodologique innovante pour la classe de l'espagnol comme langue étrangère}

Résumé. L'innovation pédagogique proposée présentée dans ce document, appelé Hour Genius est une contribution du monde des affaires en tant que stratégie que nous voulons utiliser l'apprentissage innovateur dans la salle de classe de l'espagnol comme langue étrangère (ELE). Hour Genius s'inscrit

\footnotetext{
1 Colegio Internacional, Madrid

clara.bieger@yahoo.es

2 Facultad de Educación y Salud. Universidad Camilo José Cela, Madrid

pcaballero@ucjc.edu
} 
dans la méthodologie de l'apprentissage par projet (PBL), où la recherche joue un rôle clé. Il est conçu pour être mis en œuvre dans un contexte scolaire multiculturel des élèves adolescents. Base du compte de la façon dont il peut être mis au point dans la classe de langue, attendue de la base théorique et les avantages de l'expérience professionnelle, et finit par pointer leurs moyens de continuité, parmi lesquels le test empirique de cette expérience réussie de l'entreprise et changement proposé pour l'enseignement de l'espagnol.

Mots-clés: classe ELE, heure Genius, l'innovation pédagogique, l'apprentissage basé sur la recherche (ABR), apprentissage basé sur le projet (ABP).

Sumario: 1. Introducción. 2. Fundamentación teórica. 2.1. El aprendizaje basado en investigación, el aprendizaje basado en proyectos y Genius Hour. 2.2. Genius Hour en el ámbito educativo. 3. Genius Hour en la clase de español como lengua extranjera. 3.1. Análisis crítico del contexto. 3.2. Participantes. 3.3. Objetivos. 3.4. Contenidos y competencias. 3.5. Metodología. 3.6. Actividades. 3.7. Recursos. 3.8. Evaluación. 4. Resultados y discusión. 5. Conclusiones. 6. Prospectiva. 7. Referencias bibliográficas.

Cómo citar: Bieger Morales, C.; Caballero-García, P. (2019). Genius Hour: una estrategia metodológica innovadora para la clase de Español como Lengua Extranjera, Didáctica. Lengua y literatura, 31, 11-28.

\section{Introducción}

A lo largo del tiempo, han sido muchas las teorías psicológicas que han intentado explicar los procesos por los cuales una persona aprende una segunda lengua (L2). Cada teoría ha generado distintos enfoques que, a su vez, han dado lugar a diferentes métodos. Cada método ha planteado distintos tipos de ejercicios y formas de dirigir la clase, si bien hoy son muchos los docentes que siguen un método de enseñanza ecléctico.

Así, el enfoque tradicional dio lugar en el siglo XIX al método de gramáticatraducción, basado en las teorías estructuralistas. Este método tenía como objetivo que los alumnos supieran traducir textos, para lo que se les proponían ejercicios de traducción, de huecos o de sinónimos y antónimos, muy aburridos y frustrantes, que dificultaban el intercambio comunicativo.

A mediados del siglo XIX comenzó a plantearse la necesidad de un nuevo enfoque pedagógico. Surgió así el Movimiento de Reforma, bajo el que apareció el método directo, que supuso una nueva orientación a la enseñanza de lenguas, al dar prioridad a la lengua oral y basar la enseñanza en la utilización de la L2. Este método, que toma el nombre de su creador y que se conoce como método Berlitz, sigue utilizándose hoy en día.

El método audio-lingual, surgido en Estados Unidos en 1950 y fundamentado en el conductismo verbal de Skinner (1957), utilizaba ejercicios basados en la repetición oral de enunciados, aprendiendo el alumno la L2 a través de la formulación mecánica de hábitos. Este método, daba una falsa sensación de seguridad y dificultaba la comunicación natural y fluida, ya que era imposible memorizar toda la casuística de situaciones comunicativas.

A finales de los años 60, el método audio-lingual comenzó a decaer y se llegó a la conclusión de que para aprender la L2, el estudiante debía desarrollar la competencia comunicativa y no solo la competencia lingüística (Hymes, 1971). Surgió así el enfoque comunicativo, cuyo objetivo era que el alumno fuese competente a la hora de comunicarse con un hablante nativo, para lo cual debía desarrollar la competencia 
gramatical, sociolingüística, discursiva y estratégica. Supuso una novedad: el alumno pasó a ser el protagonista del proceso de aprendizaje y el profesor su facilitador.

En 1983, Terrell y Krasen establecieron los fundamentos del enfoque natural, que toma como base el enfoque comunicativo y ve el aprendizaje de un primer y segundo idioma de forma similar, por lo que la L2 se adquiere de forma natural dentro del aula. Se trata, pues, de un enfoque en el que predomina la oralidad y el significado, por lo que no hay una enseñanza formal de la gramática ni del vocabulario.

A finales de los años 80, comenzó a proponerse una enseñanza basada en los principios constructivistas del aprendizaje, centrada en los procesos comunicativos (no en los contenidos), conocida en la enseñanza de lenguas como enfoque por tareas, equivalente al ABP de la pedagogía general. En este sentido, los proyectos se utilizan como un elemento mediador del aprendizaje, tanto del contenido objeto de estudio como de los procesos de investigación y, en el caso de la L2, de los procesos comunicativos.

Es común entre los profesores de ELE no seguir un método de forma pura, sino optar por una metodología ecléctica que permita utilizar lo que el docente considera mejor de cada método. Así, es normal el uso de ejercicios de huecos (propios del método tradicional), de solo la lengua meta (método directo), canciones para introducir una estructura gramatical (método natural), puestas en común en clase o charlas informales (enfoque comunicativo), explicaciones magistrales de un aspecto concreto de la lengua (método tradicional) o ejercicios en voz alta para practicar una estructura gramatical (método audiolingual). Y a su vez, todo esto puede formar parte de las estrategias del profesor para que sus alumnos aprendan los conocimientos de la L2 necesarios para desarrollar un proyecto (enfoque por tareas).

A pesar de esta diversidad de opciones, encontramos un factor común, el aburrimiento y la falta de motivación intrínseca, debido a que los alumnos sienten una desconexión entre lo que hacen en clase y la realidad de su vida personal y social. Esto hace necesario que, como docentes de ELE, encontremos opciones metodológicas que nos ayuden a conseguir los mejores resultados en el aprendizaje. En este sentido, el enfoque ABI y la metodología de ABP han demostrado ser útiles en el aprendizaje, además de una respuesta al cambio educativo que se le pide a la escuela.

El objetivo de este trabajo es ofrecer una nueva estrategia didáctica, llamada $\mathrm{Ge}$ nius Hour, adaptada del mundo empresarial al educativo, y de enfoque ABI, que resulta motivadora para el alumno, útil para ELE, de la que no hay referencia de uso en el aula de L2. En este sentido, consideramos que Genius Hour supone una innovación educativa y algo inédito en el aprendizaje de idiomas.

\section{Fundamentación teórica}

Genius Hour, 20\% Time Projects o Passion Projects son los diferentes nombres que se han utilizado para designar esta nueva estrategia de aprendizaje, llamada así porque permite a los alumnos emplear un tiempo determinado dentro del horario escolar (un 20\% del tiempo de la asignatura), para investigar de forma individual sobre algún tema específico que el alumno elige y le apasiona (Juliani, 2015).

Genius Hour tiene su origen en el mundo empresarial, donde grandes compañías, como Google o $3 \mathrm{M}$, dejaban a sus ingenieros un $20 \%$ de su tiempo para que investigasen aspectos de su interés, del que han nacido productos de gran éxito, como Gmail o Post-it (Krebs y Zvi, 2016). 
La integración de Genius Hour en el mundo educativo pretende cubrir el desfase que existe entre lo que la escuela ofrece y lo que un mundo globalizado y cambiante demandan. En el entorno educativo actual, donde prima el conocimiento frente a las habilidades y las actitudes, la conexión empresa-escuela es un tema especialmente interesante (Juliani, 2015). En esta conexión está un aspecto de la innovación de nuestra propuesta para el contexto educativo. en general, y para la clase de ELE, en particular.

Genius Hour se encuadra dentro la metodología ABP, en la que el alumno aprende activamente a través de preguntas desafiantes que le involucran en el diseño, la toma de decisiones y la investigación para la respuesta, permitiéndole trabajar de forma relativamente autónoma, y culminando con la presentación de un producto final (Thomas, 2000).

\subsection{El aprendizaje basado en investigación, el aprendizaje basado en proyectos y Genius Hour}

La enseñanza tradicional ha estado basada en el aprendizaje pasivo, carente de creatividad, pensamiento crítico e investigación (Morán, 2004). Cada vez es más evidente la necesidad de profesionales que sean capaces, no solo de replicar sino de crear conocimientos (Peñaherrera, Chiluiza y Ortiz, 2014). En este sentido, el ABI es un enfoque que tiene sus raíces en las teorías constructivistas y surge como un enfoque pedagógico de estrategias de aprendizaje activo para desarrollar en el estudiante competencias que le permitan realizar una investigación creativa en el mundo del conocimiento (ITESM, 2017). Estos conocimientos y su aplicación son fundamentales para mejorar la educación (Coll, 1996).

El ABI como enfoque pedagógico y el ABP como metodología derivada, tiene sus bases en el constructivismo. El alumno aprende construyendo nuevas ideas sobre las experiencias o conocimientos previos (Carretero, 1997) y diseña proyectos que le permiten construir conocimiento y tienen aplicación en la vida real (Villar, 2013).

En lo referente al aprendizaje y la investigación, Dewey (1916) proponía, a comienzos del siglo $\mathrm{XX}$, una pedagogía basada en la experiencia, que permitiera la conexión entre lo que se hace y la consecuencia que se recibe. Entendiendo la experiencia como fuente de conocimiento, se deriva que no solo hay que centrarse en la dimensión cognitiva del alumno, sino en la práctica, porque se aprende "haciendo" (Peñaherrera et al, 2014).

Piaget, por su parte, postulaba que el nuevo conocimiento se asimila por la relación con las ideas previas, incorporando los conceptos de acomodación, asimilación y adaptación, según los cuales, el aprendizaje tiene lugar cuando se modifican las estructuras cognitivas preestablecidas (Pozo, 2009). Para Piaget, el profesor debe presentar a los alumnos situaciones problemáticas que conduzcan a los estudiantes al planteamiento continuo de preguntas, como forma de reorganizar sus conocimientos y avanzar por los mismos (Cáceres y Munévar, 2016).

Y, si para Piaget (1975) el aprendizaje era algo personal (constructivismo psicológico), para Vygotsky (1979) el conocimiento era algo social (constructivismo social). En este sentido, una de las principales aportaciones de Vygotsky fue la idea de que los procesos psicológicos superiores, como el lenguaje y el razonamiento, se adquieren primero en un contexto social y después se internalizan, por lo que un proceso interpersonal queda transformado en otro intrapersonal. Su zona de desarrollo 
próximo, "no es otra cosa que la distancia entre el nivel real de desarrollo, determinado por la capacidad de resolver independientemente un problema, y el nivel de desarrollo potencial, determinado a través de la resolución de un problema bajo la guía de un adulto o en colaboración con otro compañero más capaz" (p.133).

Ausubel (1976) aportó su teoría sobre el aprendizaje significativo, según la cual el conocimiento se construye a partir de los conocimientos previos del alumno. Propuso un aprendizaje progresivo y comprensivo, considerando que lo que se comprende se puede aprender y recordar con mayor facilidad, puesto que se integra en la estructura de conocimientos del alumno (Arceo, Rojas y González, 2002). Asimismo defendió que la disposición positiva del alumno está relacionada con el logro del aprendizaje significativo (Cáceres y Munévar, 2016).

Bruner (1966) añadió que el aprendizaje no debe limitarse a una memorización mecánica de las ideas o procesos, sino que debe preparar al alumno para la resolución de las situaciones a las que se enfrenta. En el aprendizaje por descubrimiento, los alumnos construyen por sí mismos sus propios conocimientos, en contraposición a la enseñanza tradicional en la que el profesor pretende que la información sea simplemente recibida por los estudiantes (Eleizalde, Parra, Palomino, Reyna y Trujillo, 2010).

Una de las características principales del aprendizaje por descubrimiento es que el contenido no se facilita en su forma final, sino que tiene que ser descubierto por el sujeto, lo que confiere un rol activo al estudiante (Martínez y Zea, 2004), que le permitirá aplicar lo aprendido a situaciones nuevas (Bruner, 1966). Aunque existen distintos grados de autonomía en el aprendizaje por descubrimiento, en el contexto educativo actual el profesor suele actuar como orientador o guía del alumno (Woolfork, 2006).

La enseñanza por descubrimiento proporciona a los estudiantes oportunidades para buscar, explorar y analizar. Estas oportunidades no solo incrementan el conocimiento de los estudiantes acerca del tema, sino que estimulan su curiosidad y los ayudan a desarrollar estrategias para aprender a aprender (Good y Brophy, 1995). Considerando que no hay una comprensión real hasta que el alumno aplica dicho conocimiento en otras situaciones, aprender implica interpretar la situación, establecer relaciones entre los factores relevantes, seleccionar, aplicar reglas, métodos, y construir sus propias conclusiones (Bruner, 1980).

Otros autores han tratado la importancia de la indagación en el aprendizaje. Short (1996) propone una metodología ABI, fundamentada en las teorías constructivistas, cuya idea principal es que los estudiantes construyan sus conocimientos cimentados en la experiencia práctica y la investigación (Peñaherrera et al,2014). Esta indagación, en función del papel que desarrolla el alumno, puede ser abierta, guiada, estructurada y dirigida (Kitot, Ahmad y Seman, 2010). Las aportaciones de Short han inspirado los planteamientos pedagógicos de instituciones de reconocido prestigio internacional, como es el caso del International Baccalaureate Organization (IBO), cuya metodología siguen todos los colegios adscritos al mismo, que ascienden, en 2016, a 5.794 colegios, 85.708 alumnos y 2.192 universidades en todo el mundo que reconocen la validez de sus programas (IBO Annual Review, 2016).

En la misma línea, Barell $(2003,2007)$ plantea la indagación como algo fundamental en el proceso de aprendizaje y define el ABP como un proceso que sirve para resolver preguntas, curiosidades, dudas e incertidumbres sobre situaciones complejas de la vida, para lo que el estudiante debe procesar información, resolver problemas, 
experimentar y reflexionar. El ABP es una forma más completa e interdisciplinar de resolver situaciones, que la única visión de una única asignatura.

Finalmente, en su análisis de las principales metodologías de enseñanza y su importancia en el desarrollo de competencias, Fortea (2009) señala que las metodologías centradas en el estudiante (como el ABP) son especialmente adecuadas para el desarrollo de la motivación, la memoria a largo plazo, el pensamiento y la generalización de lo aprendido.

Diversos estudios sobre la eficacia de ABP en las clases de alumnos de secundaria, como los realizados por Molina (2015) y Aliane (2006) señalan que, como resultado de su aplicación, los alumnos están más implicados en las clases, motivados y participativos, son capaces de reflexionar sobre su propio aprendizaje, y alcanzan un nivel claramente mayor que en el aprendizaje tradicional.

Podemos decir, a la vista de la revisión de la literatura realizada, que tanto el ABI como el ABP tienen una sólida fundamentación pedagógica y constituyen, como señala Juliani (2015), el marco en el que se encuadra y fundamenta Genius Hour.

\subsection{Genius Hour en el ámbito educativo}

La utilización de Genius Hour en el ámbito educativo es muy reciente y la literatura rigurosa al respecto es todavía limitada. Destacan principalmente las aportaciones de Juliani (2015), Krebs y Zvi (2016) y Maiers y Sandvold (2014).

Juliani (2015) es uno de los investigadores más activos en el desarrollo práctico de esta metodología. En su obra Inquiry and Innovation in the Classroom, el autor plantea Genius Hour como una forma efectiva de preparar a los alumnos para el mundo real que les toca vivir y ofrece ideas prácticas sobre cómo aplicar esta técnica en cada uno de los niveles educativos escolares, explicando las fases de implantación y facilitando materiales para el profesor (posibles preguntas, plantillas o listas de comprobación). Aporta también casos reales de éxito y hace un análisis de las posibles dificultades de implantación en el aula, centradas en el escepticismo de padres, directores y/o educadores respecto de esta técnica.

Krebs y Zvi (2016) entienden Genius Hour como una forma de desarrollar la creatividad, la curiosidad y las ganas de aprender en los alumnos. Su obra The Genius Hour guidebook: Fostering Passion, Wonder, and Inquiry in the Classroom pretende ser una guía práctica para la implantación de esta técnica en el aula más que una reflexión metodológica. Explica los pasos a seguir, ofrece recursos y materiales para el profesor, así como actualizaciones en web de los mismos, con casos reales de éxito.

Maiers y Sandvold (2014) centran su trabajo en la importancia de descubrir la pasión de cada alumno, refiriéndose a Genius Hour como Passion-driven learning. En su libro The Passion-driven Classroom: a Framework for Teaching and Learning, las autoras definen el papel del profesor y del alumno en este tipo de proyectos y establecen tres puntos clave de trabajo en el aula: el descubrimiento de lo que apasiona a cada alumno, la puesta en práctica de la investigación y la exposición a una audiencia de lo aprendido. Como resultado de ello, los alumnos aprenden nuevas habilidades y exploran sus talentos con la ayuda del profesor, convirtiéndose en aprendientes a largo plazo, apasionados y deseosos de conocimiento.

Aunque los autores referenciados (Juliani, 2015; Krebs y Zvi, 2016; Maiers y Sandvold, 2014) aportan aspectos prácticos propios, todos coinciden en señalar cuá- 
les son los pasos a seguir en la implementación de Genius Hour en el aula: 1. Seleccionar un tema de interés para el alumno, 2. Formular una pregunta abierta, guía para llevar a cabo la investigación, 3. Planificar el trabajo, tanto en lo relativo a la investigación como al formato del producto final, 4. Buscar información y recopilar datos, analizarlos y obtener conclusiones, 5. Crear el producto final y 6. Compartirlo con una audiencia. Por lo que respecta a las reglas de funcionamiento, destacan dos: la realización de un trabajo de investigación individual, que al final se presenta a una audiencia y, la evaluación, tanto del proceso como del resultado.

La implantación de Genius Hour como estrategia de aprendizaje tiene los siguientes beneficios (Juliani, 2015; Peñaherrera et al., 2014; Villar, 2013): establece una conexión entre la realidad del mundo y la del estudiante, ayuda a los alumnos a descubrir su pasión y sus talentos, aumenta la motivación, produce un desarrollo cognitivo multidisciplinar y duradero que desarrolla habilidades para el pensamiento y el aprendizaje, y aumenta la autoestima.

Sin embargo, en la revisión de la literatura no hemos encontrado nada referente al uso de Genius Hour en la clase de idiomas, por lo que resultaría algo inédito en la enseñanza de ELE y la principal aportación de nuestra propuesta. Una opción que metodológicamente sería coherente con la propuesta del Consejo de Europa (2002) en el Marco Común Europeo de Referencia para las Lenguas (MCER).

A los beneficios de Genius Hour para el aprendizaje, añadimos los que se derivan de utilizarlo como estrategia para las clases de ELE: a) una exposición intensa y motivadora a la lengua meta, ya que el alumno entra en contacto con el español desde una perspectiva real y satisfactoria, debido a que está altamente interesado en lo que quiere aprender, al tiempo que el contacto con el idioma es muy completo, tanto por la diversidad de materiales a los que se expone como por la variedad de sus propias elaboraciones. b) una motivación intrínseca, con acceso a niveles superiores de dominio lingüístico, de acuerdo con los trabajos de Gardner y Lambert (1972) sobre la relación entre la motivación y el aprendizaje de una segunda lengua.

Fundamentado, teórica y pedagógicamente, Genius Hour como estrategia metodológica innovadora, planteamos ahora su viabilidad y efectividad en el aula de ELE, describiendo cómo ha sido implementado y valorando sus resultados.

\section{Genius Hour en la clase de español como lengua extranjera}

\subsection{Análisis crítico del contexto}

El contexto de nuestra intervención ha sido un colegio americano de Madrid, privado y mixto, que sigue el sistema americano de enseñanza y cubre todas las etapas educativas. El colegio ofrece tanto el High School Diploma como el International Baccalaureate (IB) en inglés. Sus estudiantes pueden acceder a universidades americanas y españolas. Tiene alrededor de 500 alumnos, de más de 25 nacionalidades diferentes, aunque la mayoría son españoles. Los estudiantes extranjeros representan aproximadamente el $20 \%$ del total.

"Spanish as a Second Language" es una asignatura que estudian los alumnos extranjeros de habla no hispana durante siete cursos, de $6^{\circ}$ grado a $12^{\circ}$ grado, según el sistema americano, de $6^{\circ}$ de Primaria a $2^{\circ}$ de Bachillerato, según el sistema educativo español (Ministerio de Educación, Cultura y Deporte, 2017). 
Los alumnos tienen 4 horas de clase a la semana, en cuatro periodos de 55 minutos. Las clases son obligatorias y, por esta razón, la actitud de los alumnos no siempre es positiva. Algunos han venido a España obligados por sus padres, dejando atrás a sus amigos. Otros saben que están de paso y no muestran interés ni por el idioma ni por la cultura. Son pocos los que de verdad tienen ganas de aprender el español como segunda lengua. Su nivel de inmersión lingüística es muy bajo: en el colegio hablan inglés, en casa su idioma materno (inglés, chino, georgiano, persa, portugués...) y en la calle no suelen relacionarse con españoles que no hablen inglés.

Las clases de español están organizadas por niveles: bajo, intermedio y alto, con alumnos de $6^{\circ}$ grado a $12^{\circ}$ grado en cada clase. El nivel bajo está integrado por alumnos que no saben nada de español, equivalente, según el MCER, a un nivel A.1 y en algunos casos a un A.2; el nivel intermedio está formado por alumnos que tienen un B.1 y B.2 y, el nivel alto, por alumnos de nivel C.1, con predominio de habla hispana, que llevan muchos años viviendo en países no hispanohablantes y estudiando en otros idiomas, sobre todo en inglés, por lo que presentan dificultades con el español formal (ortografía, vocabulario, comprensión lectora de determinados tipos de textos...).

Para mejorar su capacidad de comunicarse en español, los alumnos necesitan incrementar su contacto con el idioma a través de exposiciones que les resulten interesantes y motivadoras. En este sentido, Genius Hour es una opción novedosa y muy interesante, ya que, a través de la investigación sobre temas de máximo interés para ellos, los alumnos experimentan cómo el español deja de ser algo aburrido y una barrera para la comunicación, para convertirse en una puerta que se abre a nuevas experiencias y posibilidades de aprendizaje.

\subsection{Participantes}

Genius Hour se ha implementado en una clase de 11 alumnos de nivel intermedio de ELE (nivel B.2), con las siguientes características: un chico de 12 años $\left(6^{\circ}\right.$ grado- $6^{\circ}$ Primaria) de Túnez, un chico de 13 años ( $7^{\circ}$ grado- $1^{\circ}$ ESO) de Brasil, un chico de 13 años ( $7^{\circ}$ grado- $1^{\circ}$ ESO) de Gran Bretaña, un chico de 14 años ( $8^{\circ}$ grado- $2^{\circ}$ ESO) de Corea, una chica de 14 años $\left(8^{\circ}\right.$ grado- $2^{\circ}$ ESO) de Filipinas, una chica de 15 años ( $9^{\circ}$ grado- $3^{\circ}$ ESO) de Brasil, un chico de 15 años ( $9^{\circ}$ grado- $3^{\circ}$ ESO) de Georgia, un chico de 16 años $\left(10^{\circ}\right.$ grado-4 ${ }^{\circ}$ ESO $)$ de China, una chica de 16 años $\left(10^{\circ}\right.$ grado- $4^{\circ}$ ESO) de Estados Unidos, un chico de 17 años $\left(11^{\circ}\right.$ grado- $1^{\circ}$ Bachillerato) de Japón y una chica de 18 años $\left(12^{\circ}\right.$ grado-2 ${ }^{\circ}$ Bachillerato $)$ de Brasil.

Con esta mezcla de edades y orígenes, gestionar la clase para el profesor es complicado, porque los alumnos tienen necesidades muy distintas, independientemente de las características de aprendizaje propias de cada alumno.

\subsection{Objetivos}

Los objetivos generales fueron: acercar el español a los alumnos de una forma motivadora y auténtica; establecer una conexión entre la realidad y el mundo del estudiante, ayudando a los alumnos a descubrir su pasión y sus talentos y facilitando su aprendizaje en torno a ellos; facilitar un aprendizaje multidisciplinar, autónomo y duradero; y aumentar la autoestima de los alumnos.

Los objetivos específicos fueron: conseguir que los alumnos entren en contacto con una gran variedad de recursos auténticos en español (lecturas, vídeos, podcasts, 
entrevistas...) y que tengan que producir distintos tipos de textos (escritos y orales), que les ayuden a mejorar su nivel de ELE; ayudarles con las dificultades idiomáticas con las que puedan encontrarse, utilizando en clase una metodología diferente a la tradicional, como Genius Hour, centrada en sus intereses y motivaciones; conseguir que planifiquen su trabajo de aula: tiempos, recursos e hitos; guiarles en el proceso de investigación, a través de preguntas estimulantes y de la búsqueda de fuentes de información rigurosas, sobre un tema de máximo interés para ellos y de su elección; orientarles para que mejoren sus destrezas comunicativas a la hora de exponer de forma exitosa el producto final de su proyecto a una audiencia; evaluarles de forma constructiva y a lo largo de todo el proceso, para mejorar su aprendizaje y las destrezas conseguidas con el uso de la indagación como recurso educativo; e implicarles y hacerles protagonistas de su proceso de aprendizaje.

\subsection{Contenidos y competencias}

Los contenidos trabajados fueron: a) procedimentales, propios del proceso de investigación que se desarrolla con esta metodología: selección de un tema de interés para el alumno, formulación de una pregunta guía, planificación del trabajo, búsqueda de información, análisis y conclusiones, creación del producto final y presentación del mismo a una audiencia; b) conceptuales, propios del tema objeto de investigación; y c) contenidos lingüísticos, que, de acuerdo al Plan Curricular del Instituto Cervantes, comprendieron contenidos gramaticales, pragmático-discursivos, nocionales, discursivos y de aprendizaje.

Los alumnos también trabajaron competencias clave del currículo de Primaria, ESO y Bachillerato que establece el MECD (2015) como: comunicación lingüística, ya que el alumno estuvo en constante contacto con el idioma: leyendo, escribiendo, escuchando, hablando e interactuando; competencia matemática y competencias básicas en ciencia y tecnología, implícitas en las investigaciones a realizar, según las preguntas guías elegidas; competencia digital, porque la búsqueda de información se realizó principalmente a través de internet, así como la comunicación oral y escrita del resultado de la investigación, necesitó de soporte informático; aprender a aprender, cuando trabajaron la autonomía en el aprendizaje y los procesos, aspectos clave para conseguir un aprendizaje a lo largo de toda la vida; Sentido de iniciativa y espíritu emprendedor, ya que el alumno tuvo que "hacer", "crear", "inventar"... un producto final; y conciencia y expresiones culturales, porque a través del idioma y de los contenidos a investigar se entra en contacto con diferentes manifestaciones artísticas y expresiones culturales.

\subsection{Metodología}

Genius Hour se encuadra dentro la metodología del ABP. El profesor ya no tiene como objetivo principal estimular la adquisición de contenidos, sino promover el desarrollo de habilidades, tales como pensamiento crítico, autonomía, comunicación, creatividad y trabajo multidisciplinar. Guía a los alumnos para que construyan su propio conocimiento; no tiene que ser un experto en el tema elegido, pero sí tener claro el proceso a seguir (Juliani, 2015). El alumno, por su parte, se convierte en el protagonista de su proceso de aprendizaje, involucrándose en un proceso sistemático de investigación, tomando decisiones, indagando en el tema objeto de estudio y 
construyendo su conocimiento (Thomas, 2000). Se trata de ir descubriendo la respuesta a su pregunta guía, sin que el profesor le dé más información que la propia del proceso de investigación, resultando una metodología muy motivadora para el estudiante (Fortea, 2009).

La implantación de Genius Hour en el aula empieza con la selección de un tema de interés para el alumno, que se concreta en una pregunta guía a partir de la cual comienza la investigación, y culmina con un producto final que se presenta a una audiencia (Juliani, 2015; Krebs y Zvi, 2016; Maiers y Sandvold, 2014).

Las reglas fundamentales para el funcionamiento de Genius Hour en la clase de ELE son, primero, la investigación individual cuyos resultados se presentan a una audiencia, segundo, la evaluación tanto del proceso como del resultado y, por último, el uso exclusivo del español para comunicarse.

En nuestro contexto, Genius Hour se diseñó para ser desarrollada en el segundo trimestre del curso, período en el que el profesor tiene mayor conocimiento de sus alumnos y puede ayudarles mejor en su proceso de aprendizaje. El programa de intervención constó de doce sesiones de 55 minutos de duración. Las sesiones se desarrollaron en la clase de los viernes, por ser este el día en el que los alumnos necesitan más motivación, debido al cansancio de la semana.

\subsection{Actividades}

Primer dia: presentación del proyecto y selección de un tema de interés para el alumno

El proyecto comienza con una sesión de motivación, en la que se explica a los alumnos en qué consiste Genius Hour, con ejemplos. No se trata solo de que lo comprendan, sino de que sientan ganas de trabajar en ello, despertando su curiosidad. En nuestro caso, utilizamos citas inspiradoras y vídeos explicativos. Independientemente del medio utilizado hay que dejarles claro los siguientes aspectos: a) ellos son los que eligen el tema sobre el que quieren aprender, para lo cual deberán formularse una pregunta, que debe ser abierta, y de la que tendrán una respuesta justificada al final; b) para contestar a esa pregunta, deberán llevar a cabo una investigación; c) el producto final de su investigación puede tener el formato que ellos quieran: un video, un prototipo, una canción... que deberán compartir con una audiencia; d) se evaluará tanto el proceso como el producto final y e) todo el trabajo se hará en español. Debemos, asimismo, explicarles cómo debe llevarse a cabo la investigación y cómo les vamos a evaluar.

Una vez que tienen claro en qué consiste Genius Hour y para qué se les da una hora "libre" a la semana, debemos ayudarles a descubrir qué es lo que les apasiona, sobre qué quieren investigar. No debemos inclinarles hacia ningún tema, simplemente ayudarles a descubrir su pasión, guiándoles con preguntas, como por ejemplo: ¿qué te gusta hacer?, ¿a qué te gusta dedicar tu tiempo libre?, ¿con qué actividades se te pasa el tiempo volando?, ¿qué tipo de personas te resultan interesantes?... A partir de estas preguntas se les pide que concreten para la semana siguiente, su tema de investigación, aquel en el que ellos serán unos genios. Ese será el punto de partida de la segunda clase.

La motivación de los alumnos es uno de los puntos clave para el éxito. Como dice Juliani (2015), es lo que impulsa la innovación. Los resultados no serán buenos si 
tenemos que estar "tirando" de nuestros alumnos para que investiguen. Ellos deben ser el motor y el alma de su proyecto, no el profesor. Debe ser algo apasionante y motivador, para ellos.

Sin embargo, es posible que en este punto nos encontremos con el problema, especialmente si trabajamos con adolescentes, de una gran pasividad por parte de algunos de ellos, a los que no les interesa nada. La apatía puede estar causada por varias razones, entre las que destacan: baja autoestima, falta de capacidad de esfuerzo, de sentido de la responsabilidad y de madurez, situaciones que debemos intentar corregir buscando el modo de que cada alumno triunfe en algo (Sánchez-Cabrera, 2014).

\section{Segundo y tercer día: pregunta guía y validación de la misma}

Los alumnos han pensado durante la semana un tema que les interesa, por lo que en esta sesión deberán concretar la pregunta a la que tienen que dar respuesta con su investigación. A continuación se citan algunos ejemplos: ¿cómo funcionan las gafas?, ¿por qué nos olvidamos de las cosas?, ¿cómo hacer mejores saltos en baloncesto?, ¿por qué nos reímos?, ¿cómo se hace una página web?, ¿cómo funcionan los cohetes?, ¿cómo afecta la falta de sueño a la salud?, ¿cómo se escribe una canción? ¿Cómo es el código de signos?... (Juliani, 2015). La pregunta que se hagan y a la que deberán dar una respuesta, debe ser una pregunta abierta: no vale un tipo de pregunta cuya respuesta se pueda contestar simplemente leyendo una web ya que debe ser una pregunta que les haga reflexionar e investigar.

En la tercera sesión los alumnos se preparan para exponer la pregunta, que tiene que ser validada por el profesor. Esta exposición a toda la clase puede hacerse de varias maneras: power point, vídeo, póster... Nosotros proponemos hacerla mediante la técnica del Elevator Pitch, que consiste en presentar un proyecto en uno o dos minutos y sin apoyo audiovisual. Elevator Pitch viene del mundo empresarial, donde se imagina la siguiente situación: un emprendedor tiene una idea pero no tiene dinero para llevarla a cabo y necesita convencer a un inversor. Como estos son personas muy ocupadas, se supone que el que tiene la idea persigue al inversor hasta encontrarlo en el ascensor y aprovecha lo que tarda este en subir a sus oficinas en una moderna torre para contarle su idea y despertar en él su interés por la misma, en la que (esperemos) pondrá dinero. Elevator Pitch se está haciendo un hueco en el ámbito académico y encaja perfectamente con la filosofía de querer acercar la realidad laboral y económica a las escuelas, en la que se enmarca Genius Hour. También resulta muy útil para aprender español, ya que requiere de una gran capacidad de síntesis, de un vocabulario preciso y de la preparación del discurso, todo lo cual cobra relevancia ante la falta de soporte audiovisual.

Utilizando esta técnica, los alumnos presentan al profesor y a la clase su idea y su pregunta, para convencerles de que merecen una hora "libre" para investigar en su proyecto. Una vez validadas todas las preguntas guía de los alumnos, el profesor las pone en un póster en la clase, que permanece allí hasta la sesión final.

\section{Cuarto día: planificación del trabajo}

En esta sesión, el alumno planifica su trabajo, tanto en lo relativo a la investigación como al formato de su producto final. Respecto a la investigación en sí misma, con la guía del profesor, hace un esquema: partiendo de la pregunta guía, se hace preguntas 
más concretas que le ayudarán en el proceso de investigación, posteriormente revisa la bibliografía existente sobre el tema elegido, formula hipótesis, busca más información que analizará para poder contrastarlas, establece conclusiones que resuelven su pregunta guía inicial y las presenta a una audiencia a modo de producto final. Cada una de estas fases tiene un tiempo determinado, de acuerdo a la planificación temporal del trabajo.

Respecto al formato del producto final, y en función del tema de investigación, el alumno debe decidir la mejor manera de mostrar sus conclusiones: a través de un prototipo, de un vídeo, un discurso, una composición musical... Al final de todo ello, elaborará una lista de los recursos que necesita para llevar a cabo su trabajo: bibliográficos, material escolar general y material específico, cámara de vídeo... El profesor revisa con el alumno todo el proceso de planificación, acompañándole para que sea lo más realista posible y la meta sea alcanzable (Allen, 2015).

\section{Quinto día y sucesivos: investigación y preparación del producto final}

En estas sesiones el alumno ejecuta el trabajo según su planificación: partiendo de la pregunta guía, se hace preguntas más concretas que le ayudan en el proceso de investigación, posteriormente revisa la bibliografía existente sobre el tema elegido, formula hipótesis, busca más información y contrasta sus hipótesis, llega a conclusiones, da respuesta a su pregunta guía inicial y la presenta a una audiencia a través de un producto final.

Según la experiencia de los alumnos en proyectos de investigación, esta actividad se desarrollará con mayor o menor autonomía, pudiendo ser guiada, estructurada o dirigida (Kitot et al, 2010). El profesor guiará a los alumnos en la reflexión, análisis y conclusiones sobre su investigación, pero también sobre la búsqueda de información.

Los tipos de fuentes de información pueden ser muy variados; este aspecto enriquece el aprendizaje de ELE, ya que el alumno entra en contacto con diversos tipos de textos (orales o escritos), como pueden ser, siempre que cumplan ciertas normas de rigor científico, webs, videos, podcasts, libros, artículos... o incluso entrevistas que ellos mismos hagan.

Durante estos días el alumno también va preparando su producto final, que como ya hemos comentado, tiene formato libre: puede ser una presentación oral, un video, una canción, un prototipo... o cualquier otra idea que sirva para contestar a la pregunta inicial que dio lugar a su trabajo. Independientemente del formato que se le dé al producto final, su presentación a la audiencia irá acompañada de una explicación oral y de un turno de preguntas y/o sugerencias, por lo que el profesor guiará al alumno no solo con la creación del producto final, sino con los retos comunicativos que suponga la presentación del mismo a la audiencia: gramática, léxico, fórmulas de inicio y despedida, pronunciación, entonación, expresión corporal, estructura...

\section{Último día: presentación del producto final}

Los alumnos presentan a la audiencia su producto final. En la exposición oral, explican el camino que han recorrido desde el planteamiento del problema hasta su resolución y muestran los resultados de su investigación. La audiencia puede ser tan amplia como se quiera: un grupo reducido de alumnos, toda la clase, más alumnos 
de otras clases o cursos, otros profesores, un panel de expertos o incluso toda la comunidad, a través de las herramientas informáticas que existen en la actualidad (se puede incluir una grabación de la presentación en un blog, en la web del colegio, en una newsletter, en un canal de YouTube...). En nuestra experiencia práctica se hizo con toda la clase.

\subsection{Recursos}

Lo primero que necesitamos para implantar Genius Hour en la clase de ELE fue un contexto innovador, es decir, un colegio abierto a nuevas estrategias de aprendizaje y a una forma diferente de entender el currículo.

En segundo lugar, necesitamos un profesor con formación en ABI, ABP, Genius Hour y nuevas tecnologías, y ganas de asumir retos.

Asimismo, necesitamos una clase con equipamiento de ordenadores, software específico (Gmail, GoogleDrive, Word, Excel y Power Point), con conexión a internet, un proyector y una pantalla. En este tipo de proyectos, las TIC juegan un papel muy importante, no porque no se puedan hacer sin ellas, sino porque abren un mundo de posibilidades y permiten avanzar mucho más rápido.

En la primera sesión usamos, además de lo ya mencionado, una selección de citas inspiradoras para movilizar las ganas de investigar y sacar lo mejor de cada estudiante (por ejemplo, un vídeo sobre Genius Hour (https://www.youtube.com/ watch? $v=$ EJEBKPM1Ogs), y una batería de preguntas que les ayudaron a descubrir su pasión, como por ejemplo: ¿qué te gusta hacer?, ¿a qué te gusta dedicar tu tiempo libre?, ¿con qué actividades se te pasa el tiempo volando?, ¿qué tipo de personas te resultan interesantes?...

Para la segunda y tercera sesión, utilizamos un podcast que explicaba cómo hacer una presentación interesante de una idea (https://www.educaixa.com/-/como-presentar-tu-proyecto-en-publico). A continuación enseñamos a los alumnos un fragmento de video-ejemplo de un discurso persuasivo (escena de la película En busca de la felicidad, https://www.youtube.com/watch? $=4 \mathrm{EC} 3 \mathrm{mvmf9fc}$ ). Validadas todas las preguntas guía, se expusieron en clase, a la vista de los alumnos, a modo de póster, utilizando cartulinas blancas, post-its de colores y rotuladores negros.

En la cuarta sesión, elaboramos una lista de control de planificación (fases, objetivos, responsables, recursos necesarios, tiempo, control de resultados), en formato excel, compartida entre profesor y alumnos en Google Drive.

Para el quinto día y sucesivos, en los que se llevó a cabo la investigación, se utilizaron recursos diferentes (bibliográficos, material escolar general y específico, cámara de vídeo, móvil...), en función del tema objeto de estudio y del formato elegido para su producto final.

El último día, los alumnos presentaron su producto final a la audiencia (toda la clase), mediante pósteres, presentaciones de Power Point, y grabaciones de audio y video, para lo que utilizaron murales, cartulinas, ordenador, y proyector, materiales todos ellos que habían previsto y solicitado en la cuarta sesión.

\subsection{Evaluación}

En Genius Hour el concepto de evaluación se entiende como el feed-back que permite comprender al estudiante qué es lo que hace bien y en qué puede mejorar. La 
evaluación, por tanto, no se interpreta como una "nota" en un trabajo o en un proyecto, sino como algo más cualitativo. En este sentido, Juliani (2015) sugiere evaluar el trabajo y no calificarlo. La evaluación en Genius Hour está siempre enfocada a la construcción del conocimiento y se evalúa tanto el proceso como el producto final.

En nuestra clase de ELE evaluamos cinco aspectos: aprendizajes previos, el día a día del proceso, el producto final, el proceso en su conjunto y el aprendizaje del español en particular.

Los aprendizajes previos y la presentación de la pregunta guía se evaluó a través de la técnica del Elevator Pitch. Para el trabajo diario, Krebs y Zvi (2016) propusieron que en cada sesión se realice una evaluación por parte del profesor y una autoevaluación por parte del alumno. La evaluación por parte del profesor se hizo mediante charlas informales con cada alumno y la autoevaluación se desarrolló al final de cada sesión, en la que el alumno respondió a las siguientes preguntas: ¿qué puedo hacer mejor?, ¿qué hago cuando tengo un problema, soy capaz de resolverlo solo?, ¿qué he aprendido hoy?, ¿qué he creado hoy?, ¿quiero seguir investigando?, ¿he aprendido algo a partir de un error?, ¿qué ha sido lo mejor de Genius Hour hoy?, ¿cómo resumirías la sesión de hoy? Esta autoevaluación se compartió con el profesor a través de Google Drive y sirvió para guiar y mejorar el proceso de aprendizaje del alumno, tanto en lo relativo a su tema de investigación como en lo relativo al aprendizaje del español.

Para evaluar el producto final, Krebs y Zvi (2016) proponen una rúbrica en la que se valoran de 1 a 5 los siguientes aspectos: esfuerzo y dedicación, curiosidad e investigación, originalidad, motivación, reflexión y calidad de lo que se presenta. Esta rúbrica la completan el profesor, los compañeros y el propio alumno, de tal forma que se pueden cotejar los resultados. Juliani (2015), por su parte, recomienda utilizar su rúbrica, conocida como GRIT rubric (Guts, Resilience, Integrity and Tenacity) y medir dentro de ella aspectos como: actitud (coraje, audacia y asunción de riesgos), resilencia (reflexión y persistencia), integridad (honestidad, bondad y apertura mental) y tenacidad (resolución, firmeza y visión de futuro). Ambas pueden utilizarse de manera complementaria. Finalmente, para evaluar el Elevator Pitch, puede utilizarse la rúbrica de Maiers y Sandvold (2014), llamada "reflexión 5-4-3-2-1", que consiste en dedicar 5 minutos a decir 4 cosas que te gustan de la presentación, 3 cosas que se han hecho bien, 2 cosas que se pueden mejorar y 1 idea para la próxima vez. Esta evaluación la realiza el profesor, los compañeros y el propio alumno, como las anteriores, de forma que, una vez más, se pueden cotejar los resultados.

En nuestra intervención, la evaluación del aprendizaje de español fue formativa y continua. Atendió a la eficacia comunicativa y a la corrección de errores. Se realizó mediante conversaciones informales en clase entre profesor y alumno, en las que además de la eficacia comunicativa, se comprobó si el alumno estaba entendiendo (en términos de idioma) la investigación que estaba haciendo y las explicaciones del profesor; la autoevaluación diaria incluyó un resumen de cada sesión y respuestas a las preguntas ¿qué he aprendido hoy? y ¿qué he aprendido hoy de español? Con la rúbrica de Krebs y Zvi (2016), medimos el uso del español en términos de gramática, léxico, fonética y pragmalingüística. Con la rúbrica GRIT, medimos la capacidad de comprensión del alumno; y con la rúbrica de Maiers y Sandvold (2014), evaluamos su capacidad de expresión, sino también su capacidad de comprensión. Con todos estos instrumentos, tanto el profesor como el alumno contaron en todo momento con una visión clara del aprendizaje de ELE. 
El profesor, por su parte, también reflexionó sobre si Genius Hour fue una estrategia productiva en términos de aprendizaje del idioma. Dicha reflexión se plasmó en la respuesta a las siguientes preguntas: ¿la metodología es adecuada para mi perfil de alumnos y de clase?, ¿están maduros para ella?, ¿les ayuda a aprender?, ¿los recursos materiales, humanos y temporales con los que cuento, son suficientes?, ¿hemos cumplido los objetivos generales y específicos que nos marcamos?, ¿hemos trabajado todas las destrezas?, ¿he sabido gestionar el aula?, ¿la evaluación a los alumnos, refleja su aprendizaje? y ¿qué cosas se pueden mejorar? A esta reflexión, se añadió otra referida a los puntos de mejora para futuras intervenciones.

\section{Resultados y discusión}

Finalizada nuestra primera experiencia con Genius Hour en la clase de ELE, podemos decir, como Juliani (2015), Krebs y Zvi (2016), que los beneficios logrados fueron reales. Nuestros alumnos aprendieron la lengua española mediante investigación; conectaron la realidad del mundo con la suya propia; la técnica les ayudó a descubrir su pasión y sus talentos, aumentó su autoestima y su motivación, tuvieron un desarrollo cognitivo multidisciplinar, y lograron habilidades para el pensamiento y el aprendizaje, que seguro utilizarán a lo largo de toda la vida.

En línea con los trabajos de Gardner y Lambert (1972), según los cuales el aprendizaje de la L2 es mayor cuando existe motivación intrínseca, podemos afirmar que Genius Hour logró ese mismo resultado en nuestra aula. La exposición completa y motivadora a la lengua meta, como vehículo para acceder a sus áreas de interés personal, tuvo buenos resultados en términos de aprendizaje lingüístico.

Genius Hour también ayudará a los alumnos a encontrar su camino para que, cuando estén en el mundo laboral, tengan estrategias y sepan cómo ser buenos en aquello que se propongan en la vida (Juliani, 2015).

Desde nuestro punto de vista, y basándonos en nuestra experiencia como profesores de español con adolescentes, el punto más delicado de Genius Hour es la gestión por parte del alumno de la libertad que se le da para elegir, ya que esta conlleva un riesgo de fracaso que, si se produce, puede ser algo desmotivador para el estudiante. Para el verdadero éxito de esta propuesta es fundamental que el profesor les haga entender que el objetivo va más allá de su proyecto concreto: se trata de aprender español, y Genius Hour y su proyecto concreto son el medio elegido para ello.

\section{Conclusiones}

El objetivo de este trabajo de investigación era ofrecer una nueva estrategia didáctica para el desarrollo de la competencia lingüística en la clase de ELE y demostrar que es efectiva. Hemos propuesto Genius Hour como innovación, una propuesta didáctica que vincula el mundo empresarial con el académico, a partir de enfoques de aprendizaje ABI y ABP, motivadores para el alumno, y útiles para el aprendizaje de español. En nuestra revisión teórica y resultados hemos encontrado lo beneficiosa que resulta para que los alumnos descubran su pasión y sus talentos, aumente su motivación, se produzca un aprendizaje multidisciplinar y duradero, aumente su autoestima y tengan un contacto intenso y completo con la L2. Genius Hour es, además, coherente 
con los principios metodológicos del MCER (2002), y es algo inédito en la clase de segundas lenguas en general y en la de ELE en particular.

El análisis crítico del contexto ha sido clave para plantear una aplicación concreta de Genius Hour en la clase de ELE, en un contexto escolar multicultural de estudiantes adolescentes, que ayude a los alumnos a incrementar su capacidad de comunicarse en español, a través de la investigación sobre temas de máximo interés para ellos, y proporcionándoles una exposición intensa y motivadora al español.

Basándonos en las experiencias de éxito de Genius Hour que muestran autores como Juliani (2015), Krebs y Zvi (2016), así como en los estudios sobre el impacto positivo que tiene la motivación intrínseca en el aprendizaje de idiomas (Gardner y Lambert, 1972), y los resultados observados de nuestra experiencia directa, consideramos que Genius Hour en la clase de ELE puede conseguir buenos resultados en términos de aprendizaje lingüístico, ya que supone una exposición completa y motivadora a la lengua meta, siendo un vehículo para acceder a las áreas de interés personal de cada alumno. Asimismo, creemos que los beneficios de esta estrategia de aprendizaje van más allá de lo estrictamente lingüístico, y aportan al alumno herramientas y experiencias que puede utilizar a largo plazo, y transferir fuera del contexto escolar a otros ámbitos a lo largo de toda su vida personal, social y profesional.

\section{Prospectiva}

La propuesta que aquí planteamos tiene una limitación teórico-práctica, en el sentido de que, si bien todo el trabajo realizado durante un curso apunta a que los resultados que podamos esperar son satisfactorios, la realidad es que Genius Hour nunca se ha llevado a la práctica para el aprendizaje de idiomas de manera continuada, lo que hace que nos movamos en el campo de las expectativas y que no podamos demostrar empíricamente su eficacia, más allá de lo que pueda mostrarnos la observación sistemática de nuestra experiencia piloto en el aula.

Está demostrada la eficacia para el aprendizaje de Genius Hour, y la metodología de ABI y ABP que lo sustenta, pero nos queda comprobar empíricamente sus resultados, de manera que podamos afianzar nuestros primeros resultados.

La prospectiva principal de nuestro estudio sería, por tanto, continuar nuestra experiencia en cursos sucesivos y realizar un estudio cuasi-experimental con grupo control que nos permita comprobar de manera más precisa la eficacia de Genius Hour, en términos de aprendizaje de español, con una muestra lo suficientemente grande y representativa como para que los resultados que se obtengan puedan ser extrapolables a la población de estudio. De corroborarse nuestra experiencia de aula, Genius Hour se confirmaría como una opción efectiva y muy atractiva para el aprendizaje de español y con beneficios que van más allá de lo meramente lingüístico hacia el aprendizaje instrumental que nos demanda la sociedad del siglo XXI.

\section{Referencias bibliográficas}

Aliane, N. (2006): Una experiencia de ABO en una asignatura de robótica, Madrid, Universidad Europea de Madrid. 
Allen, M. (2015): “Genius Hour makes great teachers redundant. What happens when Genius Hour really works? One teacher finds out firsthand that the plan to engage students in learning has unintended consequences", en Educational Horizons, 94, 24-26.

Arceo, F., Rojas, G. y González, E. (2002): Estrategias docentes para un aprendizaje significativo: una interpretación constructivista, México, Mcgraw-Hill.

Ausubel, D. (1976): Psicología Educativa, Mexico, Trillas.

Barell, J. (2003): Developing more curious minds, Virginia, Association for Supervision y Curriculum Development.

Barell, J. (2007): Why Are School Buses Always Yellow? Teaching for Inquiry, PreK-5, California, Corwin Press.

Bruner, J. (1966): Towards a Theory of Instruction, Cambridge, MA, Harvard University Press.

Bruner, J. (1980): Investigación sobre el desarrollo cognitivo, España, Pablo del Río.

Cáceres, Z. y Munévar, O. (2016): "Evolución de las teorías cognitivas y sus aportes a la educación", en Revista actividad física y desarrollo humano [en línea], 7, disponible en internet, http://revistas.unipamplona.edu.co/ ojs_viceinves/index.php/AFDH/article/ view/2408/1207 [consulta: 29 de mayo de 2017].

Carretero, M. (1997): Constructivismo y Educación, México, Progreso.

Coll, C. (1996): "Constructivismo y educación escolar: ni hablamos siempre de lo mismo ni lo hacemos siempre desde la misma perspectiva epistemológica", Anuario de Psicología, 69, 153-178.

Consejo de Europa (2002): Marco Común Europeo de Referencia para las lenguas, Madrid, Secretaría General Técnica del MEC, Anaya e Instituto Cervantes.

Dewey, J. (1916): Democracia y educación, Buenos Aires, Losada.

Eleizalde, M., Parra, N., Palomino, C., Reyna, A., y Trujillo, I. (2010): “Aprendizaje por descubrimiento y su eficacia en la enseñanza de la biotecnología", Revista de investigación, 71, 271-290.

Fortea, M. (2009): Metodologías didácticas para la E/A de competencias, Castellón, CEFIRE.

Gardner, R. y Lambert, W. (1972): Attitudes and Motivation in Second Language Learning, Londres, Newbury Honse.

Good, T. y Brophy, J. (1995): Introducción a la Psicología del Aprendizaje. Psicología Educativa Contemporánea, España, McGraw-Hill.

Instituto Cervantes (2006): Plan curricular del Instituto Cervantes. Niveles de referencia para el español, Madrid, Instituto Cervantes, Biblioteca nueva.

Instituto Tecnológico de Estudios Superiores de Monterrey (ITESM) (2017): Aprendizaje basado en Investigación [en línea], disponible en internet, http://sitios.itesm.mx/va/dide2/ tecnicas_didacticas/abi/qes.htm [consulta: 7 de junio de 2017].

International Baccaleurate Organization (2017): International Baccaleurate Organization Annual Review 2015-16 [en línea], disponible en internet, http://www.ibo.org/globalassets/publications/ib-annual-review-2015-16-en.pdf [consulta: 30 de mayo de 2017].

Juliani, A.J. (2015): Inquiry and Innovation in the Classroom ( $1^{\mathrm{a}} \mathrm{ed}$.), Nueva York, Routledge.

Kitot, A.K.A., Ahmad, A.R., y Seman, A.A. (2010): "The effectiveness of inquiry teaching in enhancing students' critical thinking”. Procedia-Social and Behavioral Sciences, 7, 264273.

Krebs, D. y Zvi, G. (2016): The Genius Hour Guidebook: Fostering pPassion, Wonder, and Inquiry in the Classroom, Nueva York, Routledge.

Hymes, D.H. (1971): Social Anthropology and Language (1 ${ }^{\mathrm{a}}$ ed.). Londres, Routledge Library Editions. 
Maiers, A. y Sandvold, A. (2014): The Passion-driven Classroom: A Framework for Teaching and Learning, Nueva York, Routledge.

Martínez, E.R. y Zea, E. (2004): "Estrategias de enseñanza basadas en un enfoque constructivista", Revista Ciencias de la Educación, 24, 69-90.

Ministerio de Educación, Cultura y Deporte (2015): Competencias clave en el currículo de Primaria, ESO y Bachillerato [en línea], disponible en internet http://www.mecd.gob.es/ $\mathrm{mecd} /$ educacion-mecd/mc/lomce/el-Curriculo/curriculo-primaria-eso-bachillerato/competencias-clave/competencias-clave.html [consulta: 8 de junio de 2017].

Ministerio de Educación, Cultura y Deporte (2017): Estudiar en España: Sistema educativo preuniversitario en España. Tabla de equivalencias [en línea], disponible en internet http://www.mecd.gob.es/eeuu/estudiar/en-espana.html [consulta: 8 de junio de 2017].

Molina, E. (2015): Uso del aprendizaje basado en ABP como metodología para la mejora del proceso de enseñanza-aprendizaje de las ciencias en $4^{\circ}$ de la ESO, La Rioja, Universidad Internacional de la Rioja.

Morán, P. (2004): “La docencia como recreación y construcción del conocimiento. Sentido pedagógico de la investigación en el aula", Perfiles educativos, 26, 41-72.

Peñaherrera, M., Chiluiza, K. y Ortiz, A. (2014): "Inclusión del aprendizaje basado en investigación $(\mathrm{ABI})$ como práctica pedagógica en el diseño de programas de postgrados en Ecuador. Elaboración de una propuesta", Journal for Educators, Teachers and Trainers, 5, 204-220.

Piaget, J. (1975): Aprendizaje inteligente, Barcelona, Editorial Tekman books.

Sánchez-Cabrera, F. (2014): "El docente frente al reto de motivar al alumno", en Revista Iberoamericana de Producción Académica y Gestión Educativa [en línea], vol. enerojunio, disponible en internet http://www.pag.org.mx/index.php/PAG/article/viewFile/134/182, [consulta: 13 de junio de 2017].

Short, K., Schroeder, J., Laird, J., Kauffman, G., Ferguson, M.J., y Crawford, K.M. (1996): Learning together through inquiry, Maine, Stenhouse.

Short, K.G., Schroeder, J., Laird, J., Kauffman, G., Ferguson, M.J., y Crawford, K.M. (1999): El aprendizaje a través de la indagación: docentes y alumnos diseñan juntos el currículo, España, Gedisa Editorial.

Skinner, B.F. (1957): Verbal Behaviour. New York, Appleton - Century-Crofts.

Terrell, T. y Krashen, S. (1983): The Natural Approach: Language Acquisition in the Classroom, Oxford, Pergamon.

Thomas, J.W. (2000): A Review of Research on Project-based Learning, California, The Autodesk Foundation.

Villar, S. (2013): Aprendizaje basado en proyectos, Zaragoza, Universidad de Zaragoza.

Vygotsky, L.S. (1979): El desarrollo de los procesos psicológicos superiores, Buenos Aires, Grijalbo.

Woolfork, E.A. (2006): Psicología educativa, España, Pearson. 